\title{
Beyond the Optical Resolution in Living Cell: Biomedical Applications of Scanning Ion Conductance Microscopy
}

\author{
Yu. Korchev \\ Imperial College London, Division of Medicine, London W12 ONN, UK; e-mail: y.korchev@imperial.ac.uk
}

DOI: $10.1134 / \mathrm{S} 1990747809030283$

Molecular biology has advanced: we know much about the individual molecular components that make up living cells down to the level of the individual atoms. The challenge, however, is to fully understand the functional integration of these components. This requires determining how the molecular machines that make up a living cell are organized and interact together not at the atomic length scale but on a nm scale. To do this we need to develop and apply nanoscale techniques for the visualisation and quantification of cell machinery in real-time and on living cells. This will lead to detailed, quantitative models of subcellular structures and molecular complexes under different conditions for both normal and diseased cells.
This approach ultimately requires the development of novel biophysical methods. We have recently pioneered the development of an array of new and powerful biophysical tools based on Scanning Ion Conductance Microscopy that allow quantitative measurements and non-invasive functional imaging of single protein molecules in living cells. Scanning ion conductance microscopy and a battery of associated innovative methods are unique among current imaging techniques, not only in spatial resolution of living and functioning cells, but also in the rich combination of imaging with other functional and dynamical interrogation methods. These methods, crucially, will facilitate the study of integrated nano-behaviour in living cells in health and disease. 